\title{
Anti-N-methyl-D-aspartate receptor encephalitis in a patient with a 7-year history of being diagnosed as schizophrenia: complexities in diagnosis and treatment
}

\author{
This article was published in the following Dove Press journal: \\ Neuropsychiatric Disease and Treatment \\ II June 2015 \\ Number of times this article has been viewed
}

\author{
Chaohua Huang 1,2,4,* \\ Yukun Kangl,* \\ Bo Zhang' \\ Bin Li' \\ Changjian Qiu' \\ Shanming Liu' \\ Hongyan Ren ${ }^{1,2}$ \\ Yanchun Yang' \\ Xiehe Liu' \\ Tao $\mathrm{Li}^{1-3}$ \\ Wanjun Guo ${ }^{1,2}$
}

'Mental Health Center, West China Hospital, Sichuan University, Chengdu, Sichuan, People's Republic of China; ${ }^{2}$ State Key Laboratory of Biotherapy, Psychiatric Laboratory, West China Hospital, Sichuan University, Chengdu, Sichuan, People's Republic of China; ${ }^{3}$ Mental Health Education Center, Sichuan University, Chengdu, Sichuan, People's Republic of China; ${ }^{4}$ Mental Health Center, Affiliated Hospital of Luzhou Medical College, Luzhou, People's Republic of China

*These authors contributed equally to this work

Correspondence: Wanjun Guo Mental Health Center, West China Hospital, Sichuan University, Dianxin Nan Jie 28, Wuhou District, Chengdu, Sichuan 6I004I, People's Republic of China

Tel +862885423561

Fax +86288516 4019

Email guowjcn@163.com

\begin{abstract}
Anti- $N$-methyl-D-aspartate receptor (NMDAR) encephalitis is a form of autoimmune encephalitis associated with antibodies against the NR1 subunits of NMDARs. Although newonset acute prominent psychotic syndromes in patients with NMDAR encephalitis have been well documented, there is a lack of case studies on differential diagnosis and treatment of antiNMDAR encephalitis after a long-term diagnostic history of functional psychotic disorders. The present study reports an unusual case of anti-NMDAR encephalitis. The patient had been diagnosed with schizophrenia 7 years earlier, and was currently hospitalized for acute-onset psychiatric symptoms. The diagnosis became unclear when the initial psychosis was confounded with considerations of other neurotoxicities (such as neuroleptic malignant syndrome). Finally, identification of specific immunoglobulin G NR1 autoantibodies in the cerebrospinal fluid and greater effectiveness of immunotherapy over antipsychotics alone (which has been well documented in anti-NMDAR encephalitis) indicated the diagnosis of anti-NMDAR encephalitis in this case. Based on the available evidence, however, the relationship between the newly diagnosed anti-NMDAR encephalitis and the seemingly clear, long-term history of schizophrenia in the preceding 7 years is uncertain. This case report illustrates that psychiatrists should consider anti-NMDAR encephalitis and order tests for specific immunoglobulin G NR1 autoantibodies in patients presenting with disorientation, disturbance of consciousness, cognitive deficit, dyskinesia, autonomic disturbance, or rapid deterioration, even with a seemingly clear history of a psychiatric disorder and no specific findings on routine neuroimaging, electroencephalography, or cerebrospinal fluid tests in the early stage of the illness.
\end{abstract}

Keywords: anti- $N$-methyl-D-aspartate receptor encephalitis, schizophrenia, differential diagnosis, treatment

\section{Introduction}

Anti- $N$-methyl-D-aspartate receptor (NMDAR) encephalitis is a synaptic autoimmune disorder in which immunoglobulin (Ig)G autoantibodies recognize the GluN1 (also termed NR1 or NR1a) subunit of NMDARs. ${ }^{1}$ According to available studies, ${ }^{1-4}$ in most patients, development and progression of the disease occurs in well-defined clinical stages. The majority of patients (70\%) will develop a viral prodrome with headache, fever, nausea, vomiting, diarrhea, and/or upper respiratory tract symptoms. Within 5 days to 2 weeks, patients develop prominent psychiatric symptoms, which may include delusions, hallucinations, mania, agitation, changes in speech, disorganization, and seizures. Most cases progress into an unresponsive phase with catatonic features, including severe neurological features like seizures, movement abnormalities, 
autonomic instability, or hypoventilation, often requiring care in the intensive care unit. The disorder most commonly affects young women, although it has been reported in males and females of all ages ( 2 months- 85 years). ${ }^{1,5,6}$ About $50 \%$ of female patients have an underlying tumor (overwhelmingly found to be ovarian teratoma), ${ }^{7}$ but the latter is rare in children under 12 years of age and in male patients. ${ }^{1,5}$ In children, non-psychiatric symptoms, such as seizures, status epilepticus, dystonia, verbal reduction, or mutism, are usually recognized as the first symptom. ${ }^{2}$ The treatment options for anti-NMDAR encephalitis include first-line immunotherapy (steroids, intravenous immunoglobulins, plasmapheresis), second-line immunotherapy (rituximab, cyclophosphamide), and tumor removal, if present. ${ }^{5}$ Approximately $50 \%$ of patients may improve within 4 weeks of first-line treatment and tumor removal..$^{5}$ Second-line immunotherapy can be effective in up to two-thirds of patients with failed first-line therapies. ${ }^{5}$ Investigations have shown that some patients with this disorder may present with non-specific abnormalities on electroencephalography and magnetic resonance imaging (MRI) of the brain, and in cerebrospinal fluid (CSF) such as pleocytosis. ${ }^{2}$ A confirmed diagnosis of this disorder requires identification of specific $\operatorname{IgG}$ autoantibodies to the NR1 subunit of the NMDARs, especially in the CSF, as the sensitivity and specificity of NMDAR antibody testing is higher in CSF than in serum. ${ }^{1,8}$ The California Encephalitis Project, which was established in 1998 to explore the etiology of encephalitis, reports that anti-NMDAR encephalitis rivals viral infections as a cause of encephalitis within the California Encephalitis Project cohort. ${ }^{9}$ Over two-thirds of patients have prominent psychiatric symptoms or may initially seek psychiatric services. ${ }^{5,10}$ About $4 \%-6 \%$ of patients with isolated psychiatric episodes have anti-NMDAR encephalitis. ${ }^{11,12}$ However, the frequency of anti-NMDAR encephalitis in the general population as well as among psychotic patients has not been fully established. To avoid unnecessary diagnostic and treatment costs and permit more timely treatment, clinicians, especially psychiatrists, should consider this disorder while performing differential diagnoses. Here we report the case of a patient with anti-NMDAR encephalitis, who was initially admitted to a psychiatric ward for acute-onset psychiatric symptoms after being diagnosed with schizophrenia 7 years earlier.

\section{Case report}

The patient was a 25-year-old woman who was diagnosed with schizophrenia at the age of 18 years. At the initial presentation 7 years earlier, the patient was lazy, showed social withdrawal and delusions of reference, and repeatedly spoke about genital organs, but had no disturbance of consciousness. She was hospitalized in the psychiatric ward. General physical and neurological examinations and routine laboratory investigations at the time showed no notable abnormalities. She was therefore diagnosed with schizophrenia and treated with antipsychotics. After discharge, she discontinued the antipsychotics, and her symptom of repeated references to genital organs disappeared, but she continued to be socially withdrawn.

Six years earlier, she showed some improvement and could study at school. However, after 1 year, she refused to go to school and take examinations. She showed delusions of persecution and reference, and described experiencing auditory hallucinations in the form of a commentary but had no disturbance in consciousness. She was hospitalized in the psychiatric ward again. During that admission, she participated in a study on neuroimaging and cognitive function in schizophrenia, and a diagnosis of schizophrenia was made based on the Structured Clinical Interview for DSM-IV (SCID). ${ }^{13}$ A brain MRI yielded normal findings. Neuropsychological tests, including intelligence quotient tests, ${ }^{14}$ logical memory tests (immediate and delayed), trail-making test (TMT) part A, TMT part B, and six tests (pattern-recognition memory, delayed matching to sample, rapid visual information processing, spatial working memory, intra/extradimensional shift, and Stockings of Cambridge, which are described in detail at http://www.cambridgecognition.com) from the Cambridge Neuropsychological Automated Test Battery were administered to assess her cognitive function, and repeated during follow-up (Table 1). After treatment with ziprasidone $(120 \mathrm{mg} /$ day $)$ and quetiapine ( $0.1 \mathrm{~g}$ every night), her symptoms were gradually relieved, and she was discharged after 46 days.

Thereafter, she remained mostly stable and was able to work for 3 years while taking ziprasidone, until she developed insomnia 15 days before the most recent acute episode and was admitted to the psychiatric ward because of a 1-day history of psychotic symptoms. One day before the admission, she experienced a feeling of traveling through space and time, and could not recognize her parents or distinguish colors. Following abnormal and incomprehensible behavior and speech such as intermittent restlessness, excitement, and loud yelling, she shouted that she felt sad, spoke of suicide and homicide, and difficulty in falling asleep. She refused hospitalization and said that she would die in the hospital. Apart from standing and walking unstably and palmar sweating, she performed normally on general physical and neurological examinations and had no notable abnormalities 
Table I Performance on neuropsychological tests

\begin{tabular}{|c|c|c|c|c|c|c|}
\hline Test & $\begin{array}{l}\text { August 9, } \\
2009\end{array}$ & $\begin{array}{l}\text { January 29, } \\
2010\end{array}$ & $\begin{array}{l}\text { August I3, } \\
2010\end{array}$ & $\begin{array}{l}\text { March I7, } \\
2012\end{array}$ & $\begin{array}{l}\text { January I4, } \\
2015\end{array}$ & Reference values \\
\hline IQ & 114 & 114 & 116 & 115 & 118 & $>85$ (normal) \\
\hline VIQ & 115 & 114 & 115 & 115 & 116 & - \\
\hline PIQ & III & III & 114 & 112 & 116 & - \\
\hline Logical memory, immediate ${ }^{a}$ & 9 & 6 & 3 & 13 & $8^{c}$ & 23 (best) \\
\hline Logical memory, delayed ${ }^{\mathrm{a}}$ & 8 & 7 & 2 & 14 & $0^{c}$ & 23 (best) \\
\hline TMT-A, time (seconds) ${ }^{b}$ & 41.07 & 55.41 & 35 & 33 & $25.28^{d}$ & - \\
\hline TMT-B, time (seconds) ${ }^{\mathrm{b}}$ & 83.25 & 82.62 & 67 & 50 & $57.09^{d}$ & - \\
\hline DMS, percent correct ${ }^{\mathrm{a}}$ & 70 & 80 & 65 & 65 & 77.5 & 100 (best) \\
\hline DMS, percent correct (simultaneous) ${ }^{\mathrm{a}}$ & 100 & 100 & 100 & 100 & 100 & 100 (best) \\
\hline DMS, percent correct (all delays) ${ }^{\mathrm{a}}$ & 70 & 80 & 60 & 60 & $70^{e}$ & 100 (best) \\
\hline IED, stages completed ${ }^{a}$ & 9 & 9 & 9 & 9 & 9 & 9 (best) \\
\hline IED, total errors (adjusted) ${ }^{\mathrm{b}}$ & 13 & II & 8 & 12 & II & - \\
\hline PRM, percent correct, immediate ${ }^{a}$ & 92 & 92 & 92 & 92 & $83.33^{\mathrm{e}}$ & 100 (best) \\
\hline PRM, percent correct, delayed ${ }^{a}$ & 67 & 92 & 75 & 67 & $83.33^{e}$ & 100 (best) \\
\hline RVP, total false alarms ${ }^{\mathrm{b}}$ & 2 & 1 & 0 & 1 & 0 & 0 (best) \\
\hline RVP, probability of hit ${ }^{\mathrm{a}}$ & 0.81 & 0.59 & 0.78 & 0.89 & 0.96 & (best) \\
\hline SOC, problems solved in minimum moves ${ }^{\mathrm{a}}$ & 8 & 10 & 10 & 8 & 9 & 12 (best) \\
\hline SWM, between errors ${ }^{\mathrm{b}}$ & 9 & 0 & I & 4 & 0 & 0 (best) \\
\hline SWM, strategy ${ }^{\mathrm{b}}$ & 23 & 22 & 19 & 20 & 19 & 8 (best)-56 (worst) \\
\hline
\end{tabular}

Notes: ahigher is better; blower is better; ' patient performed worse than before; ${ }^{d}$ patient performed as well as the healthy control; ${ }^{\mathrm{e}}$ patient performed as well as before but worse than normal; DMS, IED, PRM, RVP, SOC and SWM are chosen from the Cambridge Neuropsychological Automated Test Battery.

Abbreviations: IQ, intelligence quotient; VIQ, verbal intelligence quotient; PIQ, performance intelligence quotient; TMT, trail-making test; DMS, delayed matching to sample; IED, intra-/extra-dimensional shift; PRM, pattern-recognition memory; RVP, rapid visual information processing; SOC, Stockings of Cambridge; SWM, spatial working memory.

on routine laboratory investigations conducted at the time of admission. Although an electroencephalogram showed mild abnormality, and brain MRI showed a small ischemic focus in the left frontal lobe, these were considered as non-specific findings. A relapse of schizophrenia was first considered, and ziprasidone $120 \mathrm{mg} /$ day along with irregular haloperidol or olanzapine were administered to control the symptoms of aggression after admission.

Two weeks later, modified electroconvulsive therapy (MECT) was administered because the psychiatric symptoms had not been relieved. However, after one MECT session, the patient's condition deteriorated, with fluctuations in consciousness, fever, sweating, carphology, orofacial dyskinetic movements, bed-wetting, visual hallucinations, amnesia, and intermittent hypermyotonia. Chest and abdominal computed tomography showed infection in the left lower lung. Blood tests showed the following: leukocyte count, $19.94 \times 10^{6} / \mathrm{L}$; creatine kinase, 2,223 IU/L; and lactate dehydrogenase, $707 \mathrm{IU} / \mathrm{L}$. Based on the clinical features, atypical neuroleptic malignant syndrome and an organic disorder of the brain were considered. MECT and antipsychotics were stopped. The patient underwent a lumbar puncture. CSF analysis revealed pleocytosis (karyocytes, $10 \times 10^{6} / \mathrm{L}$; normal, $<8 \times 10^{6} / \mathrm{L}$ ), and no remarkable abnormalities were detected on biochemical tests and smear examinations for bacteria, tubercle bacilli, and fungi. Empirical treatment with acyclovir and sodium valproate was administered. Pneumonia was considered, and antibiotics were administered. Irregular administrations of trazodone, clozapine, and clonazepam were also used to improve insomnia. After 4 weeks, the patient experienced some improvement in consciousness, excitement, and restlessness; her temperature went back to normal. However, she was still severely impaired in terms of personal orientation, memory, calculation, intermittent hypermyotonia, aggressiveness, and meaningless gropings.

A second lumbar puncture was conducted for routine CSF analysis plus investigation of autoimmune encephalitis antibodies. The routine CSF analysis showed normal results, but the tests for autoimmune encephalitis antibodies, including anti-NMDAR-Ab, anti-CASPR2-Ab, anti-AMPA1-R-Ab, anti-AMPA2-R-Ab, anti-LGI1-Ab, GABA2-R-Ab, anti-Hu, anti-Ri, anti-Yo, anti-PNMA2 (Ma2/Ta), and anti-CV2/CRMP2 (tested in Peking Union Medical College Hospital), were positive for NMDAR-Ab (specific IgG NR1 antibodies). The diagnosis was revised to anti-NMDAR encephalitis, schizophrenia, and pneumonia. The patient was then transferred to the neurology department for further treatment. After transfer, she was screened for possible tumors, with no notable findings on full-body positron emission tomography, or abdominal and urogenital 
ultrasonography. Repeat MRI of the brain yielded normal results. Beginning on day 86 of hospitalization, she was given intravenous immunoglobulin (22.5 g/day for 5 days) along with a 3 -week course of valproate $(500 \mathrm{mg}$ twice daily), quetiapine ( $0.4 \mathrm{~g}$ every night), and clonazepam ( $1 \mathrm{mg}$ every night). The disturbances of consciousness and sleep, hallucinations, and aggression were gradually relieved in 2 weeks, and she was discharged 105 days after admission, although some ideas of reference and complaints regarding impairment of short-term memory remained.

The patient is being followed up in the outpatient department, and two 5-day courses of intravenous immunoglobulin ( $25 \mathrm{~g} /$ day) were administered at 3 weeks and 3 months after the first course. Ten months after the last acute onset, she is being treated with quetiapine and valproate, generally performs well in terms of self-care and on neuropsychological tests, except for some deficit in logical memory (Table 1). She is prepared to seek employment, although residual ideas of reference occasionally occur.

\section{Discussion}

To our knowledge, this is the first case report of anti-NMDAR encephalitis with a seemingly clear, long-term diagnostic history of schizophrenia. Given the finding of specific IgG NR1 antibodies in CSF and relief of serious neuropsychiatric symptoms after the first course of intravenous immunoglobulin treatment in this patient, the diagnosis of anti-NMDAR encephalitis for the most recent acute onset could be confirmed.

Based on the available evidence, however, the relationship between the newly diagnosed anti-NMDAR encephalitis and the seemingly clear, long-term history of schizophrenia in the preceding 7 years is uncertain. Schizophrenia is a common, heterogeneous, and complex disorder, the etiology of which is mainly related to dysregulation of specific neurotransmitter systems, such as glutamatergic signaling. ${ }^{15,16}$ Since the initial description of anti-NMDAR encephalitis in $2007,{ }^{17}$ the notion that abnormalities in both autoimmunity and glutamatergic signaling can be involved in the etiology of psychosis is further supported. ${ }^{1}$ Psychiatric symptoms, including hallucinations, delusions, agitation, and thought disorder, which are common in schizophrenia, have also been observed as the most common initial presentation (68\%-80\%) of anti-NMDAR encephalitis. ${ }^{10,18}$ The overlapping psychotic symptoms of schizophrenia and anti-NMDAR encephalitis are considered to be related to NMDAR hypofunction. ${ }^{2,19}$ However, the association of anti-NMDAR encephalitis with schizophrenia is not well documented. The specific NR1a
IgG autoantibodies required for diagnosis of anti-NMDAR encephalitis are rarely detected in serum (similar to a healthy control group), and are never identified in CSF in both new-onset and chronic psychosis. ${ }^{12,18,20-22}$ Moreover, 9.9\% of patients with schizophrenia show antibody subtypes of IgA and/or IgM other than IgG against NR1a/NR2b (but not NR1a alone) in serum. ${ }^{23}$ These studies suggest that NMDAR autoimmunity might be relevant to schizophrenia, but the targets and antibody subtypes are distinct from those in antiNMDAR encephalitis. ${ }^{1}$

Because isolated psychiatric episodes, such as those observed at the onset or relapse of anti-NMDAR encephalitis in our patient, have been occasionally observed in previous studies, ${ }^{11}$ and the seemingly clear 7 -year history of schizophrenia was difficult to differentiate from repeated relapses of anti-NMDAR encephalitis. Furthermore, during the preceding 7 years, both clinical and laboratory diagnoses of schizophrenia had been established by a psychiatrist using mental status examination ${ }^{24}$ or $\mathrm{SCID}^{13}$ plus routine exclusion criteria, including physical, laboratory, and neuroimaging findings. In addition, the generally positive response to antipsychotics supported this diagnostic impression. However, a positive response to antipsychotics or spontaneous relief has also been documented in previous case reports of antiNMDAR encephalitis. ${ }^{5,12}$ In the present case, a lack of relief after stopping antipsychotic treatment, which is uncommon in schizophrenia, was observed after the first discharge. Thus, it is difficult to accurately differentiate between schizophrenia and anti-NMDAR encephalitis, especially for the preceding 7-year history, because specific IgG NR1a antibodies had not been tested for during the earlier psychotic episodes.

The effectiveness of antipsychotic medications, electroconvulsive therapy (ECT), and MECT for schizophrenia is well documented, ${ }^{25,26}$ and immunotherapy and removal of tumors, if present, are effective treatment options for anti-NMDAR encephalitis. ${ }^{5}$ The administration of multiple classes of psychotropic medications, such as benzodiazepines, conventional and atypical antipsychotics, and mood stabilizers, was documented in several case reports of antiNMDAR encephalitis. ${ }^{27,28}$ Of these, some have documented marginal success with use of atypical antipsychotics to target psychotic symptoms, ${ }^{27}$ while others have reported an exacerbation of neuropsychiatric symptoms and movement abnormalities with neuroleptic administration. ${ }^{28} \mathrm{ECT}$ for psychiatric symptoms of autoimmune encephalitis has not been well studied. Some improvement in psychotic and catatonic symptoms was reported in several anti-NMDAR encephalitis patients with inadequate responses to other 
treatments, ${ }^{27}$ but the combination of ECT with immunotherapy and plasma exchange failed in a 14-year-old girl with anti-NMDAR encephalitis who presented with typical signs and symptoms of catatonia. ${ }^{29}$ Although our patient responded positively to antipsychotics during earlier psychotic episodes, her condition deteriorated to a likely neuroleptic malignant syndrome after one MECT session during the most recent episode. Given that the MECT was conducted while the patient was treated with antipsychotic medication (ziprasidone $120 \mathrm{mg} /$ day, along with irregular haloperidol or olanzapine) and the natural progression of the disease may also account for the clinical deterioration, it is difficult to evaluate the role of MECT in the present case. However, this indicates that clinicians should be cautious when considering ECT and neuroleptics such as haloperidol for anti-NMDAR encephalitis. The concurrent use of multiple therapeutic modalities for the treatment of anti-NMDAR encephalitis in the studies reported so far makes it difficult to attribute the observed clinical outcomes to any particular treatment. ${ }^{28}$ There is a dearth of randomized controlled trials to evaluate the responses to such treatments.

Beyond the acute stage of illness, patients with antiNMDAR encephalitis take considerable time to return to their baseline function. Approximately $85 \%$ of patients who ultimately make a near-complete recovery with early and aggressive treatment have significant cognitive and behavioral abnormalities upon hospital discharge, requiring supervision and rehabilitation. ${ }^{7}$ Our patient also had serious cognitive deficits during the most recent acute stage, but showed recovery on tests for intelligence quotient, psychomotor performance (TMT), visual memory (patternrecognition memory and delayed matching to sample), attention (rapid visual information processing), working memory, executive function, and planning (spatial working memory, intra/extradimensional shift, and Stockings of Cambridge) 10 months from the onset of the most recent episode. Although she performed unsatisfactorily on the logical memory test, she may recover further, since full recovery was seen after a much longer duration in previous case reports. For instance, a school-aged girl who continued to have impaired cognitive function 18 months after admission improved 2.5 years after admission and completely recovered (normal cognition) after 4.5 years. ${ }^{30}$

Although screening for possible tumors showed no notable findings in our patient, long-term follow-up is required, since an ovarian teratoma was detected several years after recovery in a previous case. ${ }^{3}$ Dalmau et al recommend periodic screening for ovarian teratomas for at least 2 years, even if the patients have recovered from encephalitis. $^{2}$

A notable limitation of the present work is that the patient was not periodically tested for concentrations of specific antibodies during remission, although it has been reported to be useful for assessing the effects of treatment, especially in CSF. A retrospective study has shown that patients with a good outcome not only have a more rapid and greater decrease of CSF antibodies than those with a poor outcome, but also that the titer change in CSF is closely related to recurrence. ${ }^{8}$ The examination is unavailable in our hospital. Also, the Peking Union Medical College Hospital, where we sent the sample for testing, only provided a qualitative examination of the antibody.

The above limitation notwithstanding, the present case report documented the complexities of diagnosis and treatment in a patient with anti-NMDAR encephalitis after a long-term diagnostic history of schizophrenia, especially because specific IgG NR1 autoantibodies had not been detected in earlier psychotic episodes. Given that the initial presentation of antiNMDAR encephalitis usually consists of psychiatric symptoms, our case report emphasizes the need for psychiatrists to consider anti-NMDAR encephalitis and order tests for specific IgG NR1 autoantibodies, especially in patients presenting with disorientation, disturbance of consciousness, cognitive deficit, dyskinesia, autonomic disturbance, or rapid deterioration, even with a seemingly clear history of a psychiatric disorder and no specific findings on routine neuroimaging, electroencephalography, or CSF analysis in the early stage of the illness.

\section{Disclosure}

The authors report no conflicts of interest in this work.

\section{References}

1. Kayser MS, Dalmau J. Anti-NMDA receptor encephalitis, autoimmunity, and psychosis. Schizophr Res. October 25, 2014. [Epub ahead of print].

2. Dalmau J, Lancaster E, Martinez-Hernandez E, Rosenfeld MR, BaliceGordon R. Clinical experience and laboratory investigations in patients with anti-NMDAR encephalitis. Lancet Neurol. 2011;10(1):63-74.

3. Iizuka T, Sakai F, Ide T, et al. Anti-NMDA receptor encephalitis in Japan: long-term outcome without tumor removal. Neurology. 2008;70(7): 504-511

4. Peery HE, Day GS, Dunn S, et al. Anti-NMDA receptor encephalitis. The disorder, the diagnosis and the immunobiology. Autoimmun Rev. 2012;11(12):863-872.

5. Titulaer MJ, McCracken L, Gabilondo I, et al. Treatment and prognostic factors for long-term outcome in patients with anti-NMDA receptor encephalitis: an observational cohort study. Lancet Neurol. 2013;12(2): $157-165$.

6. Armangue T, Leypoldt F, Malaga I, et al. Herpes simplex virus encephalitis is a trigger of brain autoimmunity. Ann Neurol. 2014;75(2): $317-323$. 
7. Kayser MS, Dalmau J. Anti-NMDA receptor encephalitis in psychiatry. Curr Psychiatry Rev. 2011;7(3):189-193.

8. Gresa-Arribas N, Titulaer MJ, Torrents A, et al. Antibody titres at diagnosis and during follow-up of anti-NMDA receptor encephalitis: a retrospective study. Lancet Neurol. 2014;13(2):167-177.

9. Gable MS, Sheriff H, Dalmau J, Tilley DH, Glaser CA. The frequency of autoimmune N-methyl-D-aspartate receptor encephalitis surpasses that of individual viral etiologies in young individuals enrolled in the California Encephalitis Project. Clin Infect Dis. 2012;54(7):899-904.

10. Dalmau J, Gleichman AJ, Hughes EG, et al. Anti-NMDA-receptor encephalitis: case series and analysis of the effects of antibodies. Lancet Neurol. 2008;7(12):1091-1098.

11. Kayser MS, Titulaer MJ, Gresa-Arribas N, Dalmau J. Frequency and characteristics of isolated psychiatric episodes in anti-N-methyl-d-aspartate receptor encephalitis. JAMA Neurol. 2013;70(9):1133-1139.

12. Zandi MS, Irani SR, Lang B, et al. Disease-relevant autoantibodies in first episode schizophrenia. J Neurol. 2011;258(4):686-688.

13. First MB, Spitzer RL, Gibbon M, Williams JB. Structured Clinical Interview for DSM-IV-TR Axis I Disorders, Research Version, Patient Edition $(S C I D-I / P)$. New York, NY, USA: Biometrics Research, New York State Psychiatric Institute; 2002.

14. Wang Q, Cheung C, Deng W, et al. Fronto-parietal white matter microstructural deficits are linked to performance IQ in a first-episode schizophrenia Han Chinese sample. Psychol Med. 2013;43(10):2047-2056.

15. Coyle JT. NMDA receptor and schizophrenia: a brief history. Schizophr Bull. 2012;38(5):920-926.

16. Javitt DC. Twenty-five years of glutamate in schizophrenia: are we there yet? Schizophr Bull. 2012;38(5):911-913.

17. Dalmau J, Tuzun E, Wu HY, et al. Paraneoplastic anti-N-methylD-aspartate receptor encephalitis associated with ovarian teratoma. Ann Neurol. 2007;61(1):25-36.

18. Irani SR, Bera K, Waters $\mathrm{P}$, et al. N-methyl-D-aspartate antibody encephalitis: temporal progression of clinical and paraclinical observations in a predominantly non-paraneoplastic disorder of both sexes. Brain. 2010;133(6):1655-1667.

19. Moghaddam B, Javitt D. From revolution to evolution: the glutamate hypothesis of schizophrenia and its implication for treatment. Neuropsychopharmacology. 2012;37(1):4-15.
20. Rhoads J, Guirgis H, McKnight C, Duchemin A-M. Lack of anti-NMDA receptor autoantibodies in the serum of subjects with schizophrenia. Schizophr Res. 2011;129(2-3):213-214.

21. Masdeu JC, Gonzalez-Pinto A, Matute C, et al. Serum IgG antibodies against the NR1 subunit of the NMDA receptor not detected in schizophrenia. Am J Psychiatry. 2012;169(10):1120-1121.

22. Dahm L, Ott C, Steiner J, et al. Seroprevalence of autoantibodies against brain antigens in health and disease. Ann Neurol. 2014;76(1):82-94.

23. Steiner J, Walter M, Glanz W, et al. Increased prevalence of diverse $\mathrm{N}$-methyl-D-aspartate glutamate receptor antibodies in patients with an initial diagnosis of schizophrenia: specific relevance of IgG NR1a antibodies for distinction from N-methyl-D-aspartate glutamate receptor encephalitis. JAMA Psychiatry. 2013;70(3):271-278.

24. Wing J, Cooper J, Sartorius N. Measurement and Classification of Psychiatric Symptoms. London, UK: Cambridge University Press; 1974.

25. Leucht S, Cipriani A, Spineli L, et al. Comparative efficacy and tolerability of 15 antipsychotic drugs in schizophrenia: a multiple-treatments meta-analysis. Lancet. 2013;382(9896):951-962.

26. Zervas IM, Theleritis C, Soldatos CR. Using ECT in schizophrenia: a review from a clinical perspective. World J Biol Psychiatry. 2012;13(2):96-105.

27. Kuppuswamy PS, Takala CR, Sola CL. Management of psychiatric symptoms in anti-NMDAR encephalitis: a case series, literature review and future directions. Gen Hosp Psychiatry. 2014;36(4):388-391.

28. Chapman MR, Vause HE. Anti-NMDA receptor encephalitis: diagnosis, psychiatric presentation, and treatment. Am J Psychiatry. 2011; 168(3):245-251.

29. Mirza MK, Pogoriler J, Paral K, et al. Adjunct therapeutic plasma exchange for anti-N-methyl-D-aspartate receptor antibody encephalitis: a case report and review of literature. J Clin Apher. 2011;26(6): 362-365.

30. Slettedal IO, Dahl HM, Sandvig I, Dalmau J, Stromme P. Young girl with psychosis, cognitive failure and seizures. Tidsskr Nor Laegeforen. 2012;132(18):2073-2076.
Neuropsychiatric Disease and Treatment

\section{Publish your work in this journal}

Neuropsychiatric Disease and Treatment is an international, peerreviewed journal of clinical therapeutics and pharmacology focusing on concise rapid reporting of clinical or pre-clinical studies on a range of neuropsychiatric and neurological disorders. This journal is indexed on PubMed Central, the 'PsycINFO' database and CAS,

\section{Dovepress}

and is the official journal of The International Neuropsychiatric Association (INA). The manuscript management system is completely online and includes a very quick and fair peer-review system, which is all easy to use. Visit http://www.dovepress.com/testimonials.php to read real quotes from published authors. 